\title{
Diseño de panes funcionales a base de harinas no tradicionales
}

\author{
Design of functional breads based on \\ non-traditional flours
}

\begin{abstract}
Functional breads constitute an interesting alternative as a vehicle of new dietary fiber sources. Two ingredients which are obtained and used at regional levels were studied: green banana flour (GBF) (Musa acuminata var nanica) and American carob flour (CF) (Prosopis alba). Breads were made mixing these flours with wheat flour (WF): GBF/WF 50:50 and CF/WF 25:75. Proximal composition of all samples was assessed according to AOAC methods. Iron, zinc and calcium contents were determined by AAS and mineral dialyzability using an in vitro method. The studied flours as well as the breads were characterized by high dietary fiber contents (12.4 and 31.0 in GBF and CF; and 6.9 and 10.2 in the green banana and carob breads, respectively). Breads had about $22 \%$ less available carbohydrates than white bread. Mineral dialyzability increased in breads about 30 to $50 \%$, since fermentation promotes phytates degradation. The greatest effect was observed on iron dialyzability. These results indicate that these flours might be promising ingredients for use in the design of functional breads, with important levels of dietary fiber. These breads may be labeled as high fiber products according to current national regulations (minimum 6\%). The inclusion of CF contributed to higher iron and calcium levels while the use of $G B F$, due to its functional properties, allowed for a greater replacement of wheat flour.

Key words: functional breads; dietary fiber; mineral availability; green banana flour; American carob flour.
\end{abstract}

\section{INTRODUCCIÓN}

Numerosos estudios clínicos señalan la importancia del consumo de alimentos que ayuden a prevenir enfermedades crónicas no transmisibles (ECNT) como las cardiovasculares, el cáncer, la obesidad o la diabetes así como el déficit de micronutrientes $(1,2)$.

En este contexto es de destacar la importancia de los alimentos altos en carbohidratos ( $\mathrm{CHO}$ ), cuya ingesta sería beneficioso aumentar en las dietas de las poblaciones, según las recomendaciones actuales (3). Las metas nutricionales de macronutrientes se dirigen no sólo a disminuir los riesgos de desnutrición, sino también los riesgos de desarrollo de ECNT relacionadas con la alimentación. Por ejemplo, se sugiere moderar el consumo de grasa a un valor máximo de $30 \%$ de las calorías, mantener constantes las proteínas y consecuentemente aumentar las calorías derivadas de $\mathrm{CHO}$. La reunión de expertos $\mathrm{FAO} / \mathrm{OMS}$ sobre $\mathrm{CHO}$ en la nutrición humana acordó que su consumo mínimo debe ser
Ángela Zuleta (1)

María Julieta Binaghi (1)

Carola Beatriz Greco (1)

Cristina Aguirre (1)

Laura De la Casa (1)

Carmen Tadini (2)

Patricia Ana Ronayne de Ferrer (1)

(1) Cátedra de Bromatología. Facultad de Farmacia y Bioquímica. Universidad de Buenos Aires, Argentina. (2) Departamento de Ingeniería Química, Escuela Politécnica, Universidad de San Pablo, Brasil

Dirigir la correspondencia a: Profesora Angela Zuleta

Cátedra de Bromatología Facultad de Farmacia y Bioquímica Universidad de Buenos Aires, Argentina Junín 956. $2^{\circ}$ piso. 1113 Buenos Aires, Argentina Tel/Fax: 5411 4964-8242/3E-mail:azuleta@ffyb.uba.a

Este trabajo fue recibido el 24 de Octubrede 2011, aceptado con modificaciones el 28 de Diciembre de 2011 y aceptado para ser publicado el 20 de Mayo de 2012.

de un $55 \%$ de las calorías totales, aporte calórico que debe corresponder principalmente a $\mathrm{CHO}$ complejos disponibles con bajo índice glicémico (3). Esta recomendación enfatiza el consumo de alimentos que cumplan con esta condición y se distancian del marco conceptual que consideraba a todos los $\mathrm{CHO}$ complejos como poseedores de propiedades fisiológicas similares.

En consecuencia, los alimentos altos en almidones adquieren una relevancia especial, ya que contribuyen a cumplir las metas nutricionales, junto a otras recomendaciones más específicas, como son disminuir el consumo de azúcares simples y aumentar el de fibra dietética (4).

Los alimentos funcionales son aquéllos que proporcionan un efecto beneficioso para la salud más allá de su función básica nutricional. Resultan de la adición, sustitución o eliminación de ciertos componentes de los alimentos con la finalidad de reducir el riesgo de padecer enfermedades (5). De allí el interés en la búsqueda de nuevas fuentes como 
ingredientes en el desarrollo de alimentos que aporten estas características.

Los panes funcionales representan una alternativa interesante, por encontrarse el pan entre los alimentos más consumidos en muchos países. En Latinoamérica, Chile es el país de mayor consumo de pan, con valores de alrededor de $98 \mathrm{~kg} / \mathrm{hab} / \mathrm{año}$. En Argentina, los valores son cercanos a los 72 kg/hab/año (6).

Varios estudios muestran el uso de fuentes de fibra dietética en panes (7-9). Estos trabajos reflejan el interés por formular productos de consumo masivo enriquecidos con fibra dietética, ya que estos carbohidratos están asociados a la disminución del riesgo de ECNT tales como diabetes, enfermedades coronarias y del tracto intestinal; estos efectos metabólicos dependerían de las propiedades de hidratación, intercambio catiónico y tamaño de la partícula $(10,11)$.

Entre los potenciales nuevos ingredientes disponibles, la harina de plátano inmaduro ha sido considerada como un producto ideal para los países industrializados (12). Ha sido estudiada como ingrediente funcional y su consumo regular produciría beneficios para la salud $(13,14)$.

Otro ingrediente de interés es la harina de algarroba, que puede ser obtenida de los frutos de distintas especies de Prosopis, que abundan en el norte de Argentina. Estas harinas se caracterizan por contener un nivel de proteína similar al de los cereales así como por su alto contenido de fibra y de hidratos de carbono disponibles (principalmente sacarosa). También es destacable la presencia de minerales de importancia nutricional, como hierro y calcio (15).

Estas harinas constituyen ingredientes de consumo habitual en algunas poblaciones, pero su obtención y uso es aún de tipo artesanal.

El objetivo del presente trabajo fue estudiar materias primas como la harina de banana verde (Musa acuminata, var nanica) y la harina de algarroba (Prosopis alba) e incluir el uso de estas harinas en la elaboración de panes.

\section{MATERIALES Y MÉTODOS}

En este marco se estudiaron materias primas de uso corriente en poblaciones autóctonas, como la harina de banana verde (Musa acuminata, var. nanica) y la harina de algarroba (Prosopis alba).

La harina de banana verde (HBV), se obtuvo en la planta piloto de la Escuela Politécnica, USP, Brasil. Se obtuvo a partir de Musa acuminata, subgrupo Cavendish (Nanicão) procedente de Vale do Ribeira, São Paulo. La banana pelada y sumergida inmediatamente en solución al 0,1\% de ácido cítrico, a fin de prevenir el pardeamiento enzimático de la pulpa. Se cortó en rodajas, y se secó a $55^{\circ} \mathrm{C}$ hasta un peso constante, en un horno (Proctor and Schwartz, model K11556, Philadelphia, USA). Luego las rodajas fueron molidas en un molino MA 680 (Marconi, Piracicaba, Brazil) y pasadas por un tamiz US-mesh 60 sieve. La harina resultante fue envasada asépticamente y conservada a temperatura ambiente hasta su utilización.

La harina de algarroba (HA), fue provista por Geser (Grupo de Estudios Sobre Ecología Regional), procedente de la provincia de Formosa, Argentina. Fue obtenida por molienda integral de las vainas maduras de algarrobo blanco. En el proceso de obtención se alternan dos etapas de secado y dos de molienda para obtener niveles de humedad y granulometría que aseguren condiciones adecuadas para el almacenamiento (16).

Se elaboraron panes utilizando mezclas de las harinas precedentes con harina comercial de trigo 000 enriquecida
(HT) según ley N² 25630 (nivel de adición en mg/kg: 30 de hierro elemental, como sulfato ferroso; 2,2 de ácido fólico; 6,3 de mononitrato de tiamina; 1,3 de riboflavina y 13,0 de niacina).

El pan con harina de banana verde fue elaborado con una mezcla 50:50 de harina de banana verde y de harina comercial de trigo 000 enriquecida (PBV). Los ingredientes utilizados fueron $250 \mathrm{~g}$ de HT, $250 \mathrm{~g}$ de HBV, $10 \mathrm{ml}$ de aceite, $10 \mathrm{~g}$ de leche descremada, agua y levadura desecada.

El pan con harina de algarroba fue elaborado con una mezcla 25:75 de harina de algarroba y de harina comercial de trigo 000 enriquecida (PA). Los ingredientes utilizados fueron $375 \mathrm{~g}$ de HT, $125 \mathrm{~g}$ de HBV, $10 \mathrm{ml}$ de aceite, $10 \mathrm{~g}$ de leche descremada, agua y levadura desecada.

En ambos casos, la cocción se realizó en un horno de pan doméstico Moulinex OW 2000.

\section{MÉTODOS \\ 1-Composición química}

1.1-Humedad. Método AOAC 925.09b: Se determinó por desecación a $105^{\circ} \mathrm{C}$, hasta peso constante.

1.2-Cenizas. Método AOAC 923.03: Por calcinación a $550^{\circ} \mathrm{C}$, hasta la obtención de cenizas blancas.

1.3-Grasas. Método AOAC 920.39: Método Soxhlet, con extracción discontinua con éter de petróleo; luego de la evaporación del solvente se registró el peso del extracto etéreo (grasa bruta).

1.4-Proteínas. Método AOAC 960.52: Método de Kjeldahl, con tratamiento con ácido sulfúrico concentrado, luego de destilado se calcula el nitrógeno presente y se expresa como proteína bruta, previa utilización del factor de conversión correspondiente (6,25 para HBV y HA, 5,71 para HT). Para los panes se calcularon factores en base a la proporción utilizada en la mezcla. Para el PBV se utilizó el factor 5,98 y para el PA, el factor 5,84.

1.5-Fibra dietaria total. Método AOAC 991.43: Método enzimático-gravimétrico, con amilasas, proteasa y amiloglucosidasa, se determina la fibra dietaria como el residuo indigerible por los sucesivos tratamientos enzimáticos.

1.6-Carbohidratos. Cálculo por diferencia (se resta de 100 la suma de todos los macronutrientes, incluída la fibra dietaria, y la humedad).

\section{2-Dializabilidad}

La dializabilidad de los minerales (D) fue determinada por medio de un método in vitro (17) modificado (18). El procedimiento involucra una digestión enzimática en condiciones que simulan las fisiológicas. Cada muestra fue homogeneizada para facilitar su posterior análisis. Alícuotas de $50 \mathrm{~g}$ (11,5 g de muestra en 38,5 $\mathrm{ml}$ de agua desionizada) de los homogeneizados fueron incubadas con $5 \mathrm{ml}$ de una solución acuosa al 3\% de a-amilasa, durante 30 minutos a $37^{\circ} \mathrm{C}$ con agitación. Luego, el pH se ajustó a 2 con solución valorada de $\mathrm{HCl} 6 \mathrm{~N}$ y se agregaron $1,6 \mathrm{ml}$ de pepsina- $\mathrm{HCl}$

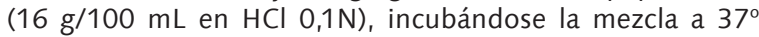
$\mathrm{C}$ durante dos horas, con agitación (proceso que simula la digestión estomacal). Dos alícuotas de $15 \mathrm{~g}$ del digerido se colocaron en erlenmeyers con bolsas de diálisis (Spectrapore Molecular Weight cut-off 6000-8000) conteniendo 18,75 $\mathrm{mL}$ de buffer PIPES 0,15 M y pH variable. El pH del buffer a utilizar fue establecido luego de hacer ensayos previos en base a la matriz alimentaria en estudio (19), para obtener un $\mathrm{pH}$ final uniforme de $6,5+0,2$, al final de la segunda incubación a $37^{\circ} \mathrm{C}$. Después de una hora de incubación, cuando el pH 
alcanzó un valor mínimo de 4,5, se agregaron 3,75 ml de una mezcla de bilis-pancreatina $(2,5 \%$ bilis y $0,4 \%$ pancreatina en $\mathrm{NaHCO} 30,1 \mathrm{~N}$ ) prosiguiéndose la incubación durante dos horas a $37^{\circ} \mathrm{C}$ para simular la digestión intestinal. Las bolsas de diálisis fueron removidas y enjuagadas con agua ultrapura y los dializados se transfirieron a tubos tarados y se pesaron. Los minerales dializados se determinaron por espectroscopía de absorción atómica $(20,21)$.

2.1-El contenido total de minerales de las muestras fue determinado en el digerido de pepsina por espectroscopía de absorción atómica previa mineralización con una mezcla HNO3 - $\mathrm{HClO} 4$ (50:50).

Para las determinaciones de Ca las muestras se diluyeron con $0,65 \%$ de lantano para suprimir la interferencia causada por los fosfatos. La dializabilidad mineral fue calculada como el porcentaje del mineral dializado con respecto a la concentración total de mineral presente en cada muestra.

Dializabilidad \% del mineral=

$$
\frac{m g \text { de mineral en el dializado }}{m g \text { de mineral en el digerido }} \times 100
$$

2.2-Se estableció el aporte potencial de cada mineral (AP) en los distintos productos teniendo en cuenta su concentración y dializabilidad.

$\mathrm{APFe}=([\mathrm{Fe}] \times \mathrm{DFe} \%) / 100 ; \mathrm{APCa}=([\mathrm{Ca}] \times \mathrm{DCa} \%) / 100 ;$

$\mathrm{APZn}=([\mathrm{Zn}] \times \mathrm{DZn} \%) / 100$

\section{Aporte calórico}

Se determinó por cálculo (22) en base a la composición de las materias primas determinada según ítem 1.

4. Análisis estadístico.

Se realizó Análisis de la Varianza (ANOVA) de un criterio y para las comparaciones a posteriori se utilizó la prueba de Tukey-Kramer con un nivel de significación global del 5\%. Se utilizó el software Infostat.

\section{RESULTADOS Y DISCUSIÓN}

En la tabla 1 se observa la composición química de las harinas de banana verde y de algarroba, así como de los panes elaborados con el agregado de estas harinas. También se incluye, con fines comparativos, la composición de la harina blanca tres ceros y la del pan blanco de harina de trigo (24).

Comparando los contenidos de nutrientes de las harinas estudiadas con la de la harina de trigo, de uso habitual en la elaboración de panes, se observa que la harina de banana verde (HBV) contiene bajos contenidos de proteína y grasa, posee un mayor contenido de fibra y cenizas y menor valor calórico. La harina de algarroba (HA) tiene una menor cantidad de proteína que la harina de trigo mientras que es mayor la cantidad de grasa, cenizas y fibra. El contenido de fibra es casi 10 veces mayor, y por consiguiente, es menor el contenido de carbohidratos disponibles, lo que resulta en una importante disminución del valor calórico, casi un 50 \% menor que el de la harina de trigo.

Este perfil de composición señala a las harinas de banana verde y de algarroba como materias primas adecuadas para el aporte de fibra.

Otros autores han reportado valores de composición de harinas de banana verde similares a los encontrados en este trabajo $(13,14,25)$. Es importante señalar que en la harina de banana verde gran parte del almidón presente es almidón resistente, lo que acrecienta el interés de esta harina como ingrediente funcional. Es necesaria la diferenciación de la etapa de madurez, ya que puede influir en sus características físico-químicas y en aspectos tecnológicos durante el procesamiento (26).

La composición de la HA estudiada se asemeja a la de otras especies de Prosopis, caracterizadas por importantes aportes de fibra dietaria y niveles moderados de proteínas si bien los contenidos absolutos pueden variar, reflejando la biodiversidad y la singularidad de cada especie (27-30).

Con respecto a la composición de los panes elaborados,

TABLA 1

Composición Química. (g /100 g en base húmeda/base seca) \pm DE

\begin{tabular}{|c|c|c|c|c|c|c|c|}
\hline Muestra & $\begin{array}{l}\text { Humedad } \\
\text { AOAC } \\
925.09 \mathrm{~b}\end{array}$ & $\begin{array}{l}\text { Grasa } \\
\text { AOAC } \\
920.39\end{array}$ & $\begin{array}{l}\text { Cenizas } \\
\text { AOAC } \\
923.03\end{array}$ & $\begin{array}{l}\text { Proteínas } \\
\text { AOAC } \\
960.52\end{array}$ & $\begin{array}{l}\text { Fibra dietaria total } \\
\text { AOAC } \\
991.43\end{array}$ & $\begin{array}{c}\mathrm{CHO} \\
\text { disponibles } \\
\text { por diferencia }\end{array}$ & $\begin{array}{c}\text { Valor } \\
\text { calórico } \\
\text { Kcal }\end{array}$ \\
\hline $\begin{array}{l}\text { Harina de banana } \\
\text { verde }(\mathrm{HBV})\end{array}$ & $6,9 \pm 0,11$ & $\begin{array}{c}0,80 \pm 0,04^{a} \\
0,86 \pm 0,04\end{array}$ & $\begin{array}{c}3,10 \pm 0,02^{a} \\
3,3 \pm 0,02\end{array}$ & $\begin{array}{l}3,60 \pm 0,19^{a} \\
3,85 \pm 0,19\end{array}$ & $\begin{array}{l}12,40 \pm 0,17^{a} \\
13,26 \pm 0,17\end{array}$ & $\begin{array}{l}73,1 \\
78,2\end{array}$ & $\begin{array}{l}314 \\
335\end{array}$ \\
\hline $\begin{array}{l}\text { Harina de } \\
\text { algarroba (HÁ) }\end{array}$ & $2,0 \pm 0,12$ & $\begin{array}{c}4,6 \pm 0.06^{b} \\
4,7 \pm 0.06\end{array}$ & $\begin{array}{l}3,20 \pm 0,03^{a} \\
3,26 \pm 0,03\end{array}$ & $\begin{array}{c}8,90 \pm 0,13^{b} \\
9,08 \pm 0,13\end{array}$ & $\begin{array}{c}31,00 \pm 0,14^{b} \\
31,62 \pm 0,14\end{array}$ & $\begin{array}{l}50,0 \\
51,0\end{array}$ & $\begin{array}{l}201 \\
205\end{array}$ \\
\hline $\begin{array}{l}\text { Harina de } \\
\text { trigo* }(\mathrm{HT})\end{array}$ & 12,7 & $\begin{array}{l}2,60 \\
2,99\end{array}$ & $\begin{array}{l}0,36 \\
0,41\end{array}$ & $\begin{array}{l}10,20 \\
11,73\end{array}$ & $\begin{array}{l}3,30 \\
3,79\end{array}$ & $\begin{array}{l}70,6 \\
81,2\end{array}$ & $\begin{array}{l}348 \\
400\end{array}$ \\
\hline $\begin{array}{l}\text { Pan banana } \\
\text { verde (PBV) }\end{array}$ & $36,3 \pm 0,18$ & $\begin{array}{l}1,60 \pm 0,05^{c} \\
2,51 \pm 0,05\end{array}$ & $\begin{array}{c}2,30 \pm 0,08^{b} \\
3,61 \pm 0,08\end{array}$ & $\begin{array}{l}4,70 \pm 0,11^{c} \\
7,69 \pm 0,11\end{array}$ & $\begin{array}{l}6,90 \pm 0,12^{c} \\
10,83 \pm 0,12\end{array}$ & $\begin{array}{l}48,2 \\
75,4\end{array}$ & $\begin{array}{l}226 \\
355\end{array}$ \\
\hline $\begin{array}{l}\text { Pan algarroba } \\
\text { (PA) }\end{array}$ & $29,7 \pm 0,13$ & $\begin{array}{c}3,60 \pm 0,09^{d} \\
5,14 \pm 0,09\end{array}$ & $\begin{array}{c}2,60 \pm 0,07^{b} \\
3,71 \pm 0,07\end{array}$ & $\begin{array}{l}5,20 \pm 0,09^{d} \\
7,99 \pm 0,09\end{array}$ & $\begin{array}{l}10,20 \pm 0,16^{d} \\
14,57 \pm 0,16\end{array}$ & $\begin{array}{l}48,6 \\
68,9\end{array}$ & $\begin{array}{l}248 \\
354\end{array}$ \\
\hline $\begin{array}{l}\text { Pan blanco* } \\
\text { (PB) }\end{array}$ & 29,8 & $\begin{array}{l}0,7 \\
1,0\end{array}$ & $\begin{array}{l}1,4 \\
2,0\end{array}$ & $\begin{array}{c}8,4 \\
12,0\end{array}$ & $\begin{array}{l}2,8 \\
4,0\end{array}$ & $\begin{array}{l}57,0 \\
81,4\end{array}$ & $\begin{array}{l}268 \\
382\end{array}$ \\
\hline
\end{tabular}

Letras distintas en la misma columna indican diferencia significativa con $p<0,05$.

* Referencia 23. 
se reflejan las características de los ingredientes utilizados. El pan PBV, con un reemplazo del $50 \%$ de HT por HBV, posee mayor cantidad de cenizas y menor concentración de proteínas y valor calórico, que el pan blanco de referencia. El mayor contenido de grasa se debe al agregado de aceite como ingrediente en la elaboración del pan. Por su parte, el pan PA, con $25 \%$ de HA y $75 \%$ de HT, posee mayor cantidad de grasa y de cenizas y menor de proteínas, que el pan blanco de referencia.

Dado que el contenido de fibra dietaria total (FDT) de las HBV y HA es mucho mayor que el de la harina de trigo, su adición a los panes se refleja en un aumento proporcional en el contenido de fibra. Los contenidos de FDT de los panes elaborados con HBV y HA duplican y triplican, respectivamente, al del pan blanco. Este es uno de los aportes nutricionales más importantes de estas harinas a los panes en estudio.

Los panes elaborados a partir de estas harinas se destacan por un menor tenor de carbohidratos disponibles en comparación con el del pan blanco común (48\% vs 57\%). Por otra parte, su contenido de fibra superior al $6 \%$ permite su rotulación como de alto contenido de fibra, según el Código Alimentario Argentino. Esta situación se refleja también en el menor valor calórico de los productos más ricos en fibras.

Además de la elaboración de panes con $\operatorname{HBV}(13,31)$, también se ha planteado el desarrollo de otros productos utilizando derivados de banana verde o HA tales como barras de cereales y galletitas (32-34).

Como ya se mencionó, ambas harinas, HBV y HA, poseen un mayor contenido de cenizas que la HT, casi el triple. En la tabla 2 se observa la concentración de hierro, zinc y calcio de las harinas HBV y HA y de los panes elaborados con el agregado de estas harinas. También se incluye, con fines comparativos, la composición de la harina blanca tres ceros y la del pan blanco de HT (24).

Respecto a la concentración total de los minerales estudiados, se observa que los productos de algarroba son los que tienen los valores más elevados. Estos resultados son coincidentes con los de otros autores que señalan un importante aporte de calcio y de hierro (además de potasio y magnesio) en productos derivados del algarrobo blanco así como en otras especies de Prosopis (27-29, 34, 35).

Por el contrario, los productos de banana verde analizados no son ricos en estos minerales. Estos valores podrían variar dependiendo de muchos factores, tales como la variedad, el grado de maduración, el tipo de suelo, el clima, entre otros. En particular, la zona de cultivo sería importante ya que el contenido mineral de los suelos parece tener una gran influencia en el contenido mineral de plátanos. Sin embargo, el aporte de hierro y zinc no resulta significativo, a diferencia de lo que sí ocurre con el potasio, magnesio, cobre y boro (36). No se encontró diferencia significativa entre la harina y el pan para el zinc en los productos de algarroba ni en los de plátano, a diferencia de lo observado para el calcio y el hierro $(p<0,05)$. En el calcio se observó un menor valor, mientras que para el hierro la contribución de la harina de trigo enriquecida resultó en un incremento de su aporte.

En la tabla 3 se observa la dializabilidad de hierro, zinc y calcio de las harinas HBV y HA y de los panes elaborados con el agregado de estas harinas. La dializabilidad es un indicador de la biodisponibilidad potencial. Comparando con valores de dializabilidad de hierro de bibliografía relativos a la harina de trigo (37), los de las HA y HBV fueron más bajos que los de la

TABLA 2

Concentración de $\mathrm{Fe}$, Zn y Ca en materias primas y panes.

\begin{tabular}{|c|c|c|c|}
\hline Muestra & Fe mg/100g & Zn mg/100g & Ca mg/100g \\
\hline Harina de Algarroba (HA) & $5,01 \pm 0,58^{c}$ & $1,70 \pm 0,80^{b}$ & $141,3 \pm 2,73^{d}$ \\
\hline Pan 75:25 HT:HA & $6,63 \pm 0,21^{c}$ & $1,48 \pm 0,31^{\mathrm{b}}$ & $85,5 \pm 2,17^{c}$ \\
\hline Harina de Banana Verde (HBV) & $0,19 \pm 0,02^{a}$ & $0,22 \pm 0,03^{a}$ & $16,31 \pm 0,51^{b}$ \\
\hline Pan 50:50 HT:HBV & $0,46 \pm 0,09^{b}$ & $0,27 \pm 0,02^{a}$ & $12,41 \pm 1,02^{a}$ \\
\hline Harina de trigo $(\mathrm{HT})^{*}$ & 3,00 & 1,80 & 11,00 \\
\hline Pan blanco* & 1,00 & 1,80 & 19,00 \\
\hline
\end{tabular}

Letras distintas en la misma columna indican diferencia significativa con $p<0,05$.

* Referencia 23.

TABLA 3

Dializabilidad de Fe, Zn y Ca en materias primas y panes.

\begin{tabular}{lccc}
\hline Muestra & D\%Fe & D\%Zn & D\%Ca \\
Harina de algarroba & $3,4 \pm 0,6^{\mathrm{a}}$ & $17,6 \pm 0,8^{\mathrm{a}}$ & $19,9 \pm 1,8^{\mathrm{a}}$ \\
Pan 75:25 HT:HA & $5,1 \pm 0,2^{\mathrm{a}}$ & $26,8 \pm 0,9^{\mathrm{b}}$ & $24,3 \pm 1,2^{\mathrm{a}}$ \\
Harina de banana verde (HBV) & $6,3 \pm 0,9^{\mathrm{ab}}$ & $28,1 \pm 1,7^{\mathrm{bc}}$ & $24,6 \pm 1,6^{\mathrm{a}}$ \\
Pan 50:50 HT:HBV & $8,6 \pm 0,9^{\mathrm{b}}$ & $32,1 \pm 2,0^{\mathrm{c}}$ & $20,3 \pm 1,7^{\mathrm{a}}$ \\
\hline
\end{tabular}

Letras distintas en la misma columna indican diferencia significativa con $p<0,05$. 
harina de trigo $(\mathrm{D} \% \mathrm{Fe} 9$,9) probablemente debido al mayor contenido de polifenoles, que son importantes inhibidores de la absorción de este mineral. La misma situación se observó comparando los valores de ambos panes en relación con un pan de harina de trigo (D\%Fe 15,2).

Por otra parte, al comparar cada harina con su respectivo pan se evidenciaría el efecto beneficioso de la fermentación, dado que en ambos panes los valores de D\%Fe tienden a ser más elevados. Este mismo efecto se observa en el caso de la $\mathrm{D} \% \mathrm{Zn}$, donde la diferencia es significativa en los productos de algarroba $(p<0,05)$. Esto se debe a que durante la fermentación se degradan los fitatos, importantes inhibidores de la absorción de hierro y el zinc, que se encuentran presentes en estas matrices. Respecto de las harinas, se observa que la $\mathrm{HBV}$ es la que tiene mayor dializabilidad de zinc; en este caso, la presencia de ácido cítrico agregado durante su obtención actuaría como un promotor.

Para el caso del calcio, no hubo diferencias significativas entre los productos de algarroba y banana verde.

En la tabla 4 se observa el aporte potencial de hierro, zinc y calcio de las harinas HBV y HA y de los panes elaborados con el agregado de estas harinas. El aporte potencial toma en consideración tanto el contenido mineral como la dializabilidad. Los productos de algarroba son los que mayor valor tienen a expensas de un elevado contenido mineral y una dializabilidad moderada, mientras que los productos de banana verde son los de menor aporte, debido tanto a su baja concentración mineral como a una dializabilidad intermedia.

Se puede observar, en general, que los panes poseen mayor aporte potencial de hierro y zinc que sus respectivas harinas, como consecuencia de su mayor dializabilidad. Como ya se mencionó, este aumento puede explicarse en parte por la degradación de los fitatos durante la fermentación. En los panes con harinas de banana verde o algarroba, el aumento en la dializabilidad se debería además a que los componentes inhibidores de la absorción (principalmente polifenoles) se encontrarían en menor concentración, debido a la mezcla realizada con harina de trigo. El aporte potencial de calcio, por el contrario, fue menor en los panes.

En los productos de panificación es posible aumentar la dializabilidad de los minerales mediante el agregado de promotores de la absorción, tales como ácido ascórbico o ácido cítrico, los que han demostrado su efectividad en productos elaborados con derivados de Prosopis alba y ruscifolia (34, 35, 39).

Los panes elaborados presentaron un adecuado volumen y formación de miga y un color más oscuro que el pan blanco.

La evaluación sensorial, en forma preliminar, arrojó alto grado de aceptación, por lo que se propone realizar un ensayo de la misma de acuerdo al protocolo marcado para estos estudios.

\section{CONCLUSIONES}

Estos resultados señalan a estas harinas como una fuente interesante para su utilización en el diseño de panes funcionales, aumentando el aporte de fibra y de minerales. La harina de banana verde, por sus características funcionales, permitió un mayor reemplazo de harina de trigo que la de algarroba.

\section{RESUMEN}

Los panes funcionales representan una alternativa interesante como vehículo de nuevas fuentes de fibra. Se estudiaron materias primas cuya obtención y uso es aún de tipo artesanal: la harina de banana verde (HBV) (Musa acuminata var nanica) y la harina de algarroba (HA) (Prosopis alba). Se elaboraron panes en mezcla con harina de trigo (HT): HBV/HT 50:50 y HA/HT 25:75. La composición centesimal de todas las muestras se realizó según AOAC. Se determinó el contenido de hierro, zinc y calcio por EAA y la dializabilidad mineral por un método in vitro. Tanto las materias primas como los panes se caracterizaron por un alto contenido de fibra $(12,4$ y 31,0 para HBV y HA y 6,9 y 10,2 para los panes de harina de plátano y algarroba respectivamente). Los panes presentaron alrededor de $22 \%$ menos de carbohidratos disponibles que el pan blanco. La dializabilidad de minerales se incrementó en los panes entre un 30 y un $50 \%$ ya que la fermentación promueve la degradación de los fitatos. El mayor efecto se observó sobre la dializabilidad de hierro. Estos resultados señalan a estas harinas como ingredientes promisorios para su utilización en el diseño de panes funcionales, con un importante aporte de fibra dietaria, que permite considerarlos alimentos ricos en fibra, de acuerdo a la legislación vigente (Código Alimentario Argentino) que señala un mínimo de 6\% para dichos productos. La inclusión de HA contribuyó a un mayor aporte de hierro y calcio, mientras que la de HBV, por sus características funcionales, permitió un mayor reemplazo de harina de trigo.

Palabras clave: panes funcionales; fibra dietaria; disponibilidad de minerales; harina de banana verde; harina de algarroba.

Agradecimientos: El presente trabajo se ha realizado dentro de la Programación UBACyT, Proyectos B063, 20020100100166 y 20020090200037.

\section{BIBLIOGRAFÍA}

1. WHO 2005. World Health Organization. Global NCD Risk Factor Surveillance Strategy. Disponible en: http://www.

TABLA 4

Aporte potencial de $\mathrm{Fe}$, Zn y Ca en materias primas y panes.

\begin{tabular}{lccc}
\hline Muestra & APFe & APZn & APCa \\
& & & \\
Harina de algarroba & $0,17 \pm 0,06^{\mathrm{c}}$ & $0,31 \pm 0,03^{\mathrm{b}}$ & $28,1 \pm 1,8^{\mathrm{c}}$ \\
Pan 75:25 HT:HA & $0,34 \pm 0,02^{\mathrm{d}}$ & $0,42 \pm 0,04^{\mathrm{c}}$ & $20,8 \pm 1,2^{\mathrm{b}}$ \\
Harina de banana verde (HBV) & $0,01 \pm 0,00^{\mathrm{a}}$ & $0,06 \pm 0,01^{\mathrm{a}}$ & $4,0 \pm, 1^{\mathrm{a}}$ \\
Pan 50:50 HT:HBV & $0,04 \pm 0,00^{\mathrm{b}}$ & $0,09 \pm 0,02^{\mathrm{a}}$ & $2,5 \pm 0,7^{\mathrm{a}}$ \\
\hline
\end{tabular}

Letras distintas en la misma columna indican diferencia significativa con $p<0,05$. 
who.int/ncd/surveillance/ Consulta: marzo de 2011

2. WHO 2002. World Health Organization. Informe sobre la salud en el mundo. Reducir los riesgos y promover una vida sana. Disponible en: http://www.who.int/whr/2002/es/ index.htm Consulta: marzo de 2011.

3. FAO/WHO. 2003. World Health Organization / Food and Agriculture Organization. Diet, Nutrition and the Prevention of Chronic Diseases. WHO Technical Report Series 916. WHO. Geneva. 2003; p147-49.

4. Stubbs J, Mazian N, Whybrow S.Carbohydrates, appetite and feeding behavior in humans. J Nutr 2001; 131: 2775S-2781S.

5. Roberfroid MB. Defining functional foods, en Functional foods: Concept to product, Ed: Gibson and Williams, CRC Press, 2000.

6. Ronayne P, Brites $C$, Ferrero C, Arocha M, León AE. Capítulo 6: Efecto de los tratamientos tecnológicos sobre la calidad nutricional y saludable de panes y productos de panadería, en "Aspectos nutricionales y saludables de los productos de panificación", CYTED, Lutz M, León, A, editores. Universidad de Valparaíso Editorial, Chile, 2009, 124 - 50.

7. Almazán AM. Effect of cassava flour variety and concentration on bread loaf quality. Cereal Chem 1990; 67: 97-9.

8. Pacheco E, Cedres M, Alvarado A, Cioccia A. Substitución del afrecho de trigo por harina de almendra desgrasada de palma aceitera como fuente de fibra dietética en la elaboración de galletas y pan. Arch Latinoamer Nutr 1994; 44: 122-5.

9. Granito M, Guerra M. Uso del germen desgrasado de maíz en harinas compuestas para panificación. Arch Latinoam Nutr 1995; 45: 322-8.

10. Anderson J, Smith B, Gustafson J. Health benefits and practical aspects of high fiber diets. Am J Clin Nutr 1994; 59: 12425-75.

11. Jenkins DJ, Kendall WC, Vuksan W. Viscous fibers, health claims and strategies to reduce cardiovascular disease risk. Am J Clin Nutr 2000; 71: 401-12.

12. Cordenunsi, B.R., Lajolo, F.M. Starch breakdown during banana ripening - Sucrose synthase and sucrose-phosphate synthase. J Agric Food Chem 1995; 43, 347-51.

13. Juarez-Garcia E, Agama-Acevedo E, Sayago-Ayerdi SG, Rodriguez-Ambriz SL, Bello-Perez LA. Composition, Digestibility and Application in Breadmaking of Banana Flour. Plant Food Hum Nutr 2006; 61: 131-7.

14. Aurore $G$, Parfait B, Fahrasmane L. Bananas, raw materials for making processed food products. Trends Food Sci Tech 2009; 20:78-91.

15. Astrada E, Caratozzolo M, Blasco C, Quiroga L, Ronayne $P$, Vigilante J. Potencialidad alimentaria del bosque nativo del Chaco argentino: una experiencia prometedora basada en la harina de algarroba (Prosopis alba). Trabajos del IV Congreso Internacional ALFATER: "Alimentación, Agricultura Familiar y Territorio". 2008. (editado en CD) ISBN: 978-987-521-328-9.

16. GESER (Grupo de estudios sobre Ecología Regional). Harina de algarroba: Un alimento especial. Disponible en: http:// WWw.geser.org.an Consulta: Marzo 2007.

17. Miller D, Schrinken B.R, Rassmussen R.R. An in vitro method for estimation of iron availability from meals. Am J Clin Nutr 1981; 34:248-56.

18. Wolfgor R., Drago S.R., Rodriguez V., Pellegrino N., Valencia M.E. In Vitro measurement of iron availability in fortified foods. Food Res Int 2002; 35: 85-90.

19. Drago Silvina R., Binaghi Maria J., Valencia Mirta E. Effect of gastric digestion $\mathrm{pH}$ on iron, zinc, and calcium dializability from preterm and term starting infant formulas. $J$ Food Sci 2005; 70:(2) 107-12.

20. Official Method of Analysis of AOAC International. 17th Edition. Washington DC, USA. Association of Official Analytical Chemists. 2000.

21. Perkin-Elmer Corp. Calcio, Hierro y Zinc. Analytical Method for Atomic Absorption Spectrophotometry. Norwolk Cl. 1971.

22. Livesey G. Metabolizable energy of macronutrients. Am J Clin Nutr 1995; 62 (suppl):1135S-42S.

23. Winer BJ, Brown DR, Michels KM. Cap. III. En: Statistical principles in experimental design. $3^{\circ}$ ed. New York: Mc Graw Hill. 1991; p. 74 - 215.

24. ARGENFOODS. Base de Datos de Composición de Alimentos. Universidad Nacional de Luján. Disponible en: http:// www.unlu.edu.ar/ argenfood/Tablas/Tabla.htm Consulta: 9/05/2011.

25. da Mota, R. V., Lajolo, F. M., Ciacco, C., \& Cordenunsi, B. R. Composition and functional properties of banana flour from different varieties. Stärke. [Starch], 2000; 52 (2-3), 63-8.

26. Tribess TB, Hernandez-Uribe JP, Mendez-Montealvo MGC, Menezes EW, Bello-Perez LA, Tadini CC. Thermal properties and resistant starch content of green banana flour (Musa cavendishii) produced at different drying conditions. LWT - Food Sci Technol 2009; 42:1022-5.

27. Bravo L, Grados N, Saura-Calixto F. Composition and Potential Uses of Mesquite Pods (Prosopis pallida L): Comparison with Carob Pods (Ceratonia siliqua L). J Sci Food Agric 1994; 65: 303-6.

28. Prokopiuk D, Cruz G, Grados N, Garro O, Chiralt A. Estudio comparativo entre frutos de Prosopis alba y Prosopis pallida. Multequina 2000; 9: 35-45.

29. FAUBA, Informe Técnico 2005. Citado en Carenzo S, Rescia Perazzo A, Caratozzolo M, Astrada E, Blasco C, Quiroga L. Activación de recursos agroalimentarios desde la identidad local: La experiencia de la Algarroba en el Chaco Argentino. Trabajos del III Congreso Internacional de la Red SIAL. España, 2006.

30. González Galán A, Duarte Corrêa A, Patto de Abreu CM, Piccolo Barcelos MF. Caracterización química de la harina del fruto de Prosopis spp. procedente de Bolivia y Brasil. Arch Latinoamer Nutr 2008; 58: 309-15.

31. Pacheco-Delahaye E, Testa G. Evaluación nutricional, física y sensorial de panes de trigo y plátano verde. INCI, 2005;30,5, p.300-304. ISSN 0378-1844.

32. Ferreira Dos Santos J. Avaliação das propriedades nutricionais de barras de cereais elaboradas com farinha de banana verde. Dissertação para obtenção do titulo de Mestre. Faculdade de Ciências Farmacêuticas, Universidade de São Paulo, Brasil, 2010.

33. Estévez AM, Escobar B, Ugarte V. Utilización de cotiledones de algarrobo (Prosopis chilensis (Mol) Stuntz) en la elaboración de barras de cereales. Arch Latinoamer Nutr 2000; 50: 148-51.

34. Bernardi C, Drago S, Sabbag N, Sanchez $H$, Freyre $M$. Formulation and Sensory Evaluation of Prosopis alba (Algarrobo) Pulp Cookies with Increased Iron and Calcium Dialyzabilities. Plant Food Hum Nutr 2006; 61: 39 - 44.

35. Bernardi C. Aportes nutricionales de la harina de vinal (Prosopis ruscifolia). Disponibilidad de hierro y factores que la afectan. Tesis de Maestría, Facultad de Ingeniería Química, Universidad Nacional del Litoral, Santa Fe, Ar- 
gentina, 2000.

36. Hardisson A, Rubio C, Baez A, Martin M, Alvarez R, Diaz $E$. Mineral composition of the banana (Musa acuminata) from the island of Tenerife. Food Chem 2001; 73: 153-61.

37. Foster,M. Rodriaguez, M, Darias, M. and Diaz Romero, C. Statistical Differentiation of Bananas According to Their Mineral Composition. J Agric Food Chem 2002; 50, 6130-5.
38. Dyner L, Drago S, Piñeiro A, Sánchez $H$, González $R$ y Valencia ME. Composición y Aporte Potencial de Hierro, Calcio y Zinc de panes y fideos elaborados con Harinas de Trigo y Amaranto. Arch Latinoamer Nutr 2007; 57:69-77.

39. Bernardi C, Freyre M, Sambucetti ME, Pirovani ME. Use of Ascorbic and Citric Acids to Increase Dialyzable Iron From Vinal (Prosopis ruscifolia) Pulp. Plant Food Hum Nutr 2004; 59: 175-9. 